\title{
Attractive Quality and Creative Management of Dongyang Wood Carving
}

\author{
Qian Zheng ${ }^{1}$ \\ Institute of of Art and Design \\ Jinhua Polytechnic \\ Jinhua, China \\ e-mail: zhengqian_jh@hotmail.com
}

\author{
Zhixin Chen ${ }^{2}$ \\ School of Management \\ Zhejiang University \\ Hangzhou, China \\ e-mail: sunychen@zju.edu.cn
}

\begin{abstract}
- during the process of industrialization, there is a homogenous tendency in Dongyang wood carving because of mechanized production and Dongyang wood carving has gradually lost its unique artistic charm. Using the attractive quality theory, the paper analyzes the quality elements of Dongyang wood carving and comes to a conclusion that, expect the quality elements that can reflect the practical values, such as materials, attractive quality elements that are attached to the practical values, such as carving art and culture, shall be explored to the greatest extent; the paper also believes that creative management is an important way to improve the attractive quality of Dongyang wood carving, furthermore, the paper proposes specific measures to strengthen the creative management of Dongyang wood carving enterprises.
\end{abstract}

Keywords-Dongyang Wood Carving; Attractive Quality; Creative Management

\section{INTRODUCTION}

Dongyang wood carving is a kind of planar carving-based decorative relief. Originated in Han Dynasty, developed in Tang and Song Dynasty and flourished in Ming and Qing Dynasty, Dongyang wood carving has a history of more than one thousand years. Dongyang wood carving is known as its distinctive carving techniques and unique artistic characteristics, being one of the four types of wood carving in China. After the reform and opening up, Dongyang wood carving has gradually turned to the development path of marketization and industrialization, and the advantages of specialized market become more evident and the industrialization scale is constantly expanding. A specialized and distinctive crafts street with Dongyang wood carving as its main products was built in 1998 and Dongyang World Trade City Craft Specialized Market was built in 2007. As of 2010, there are nearly 1000 arts and crafts enterprises and more than 2000 family workshops in Dongyang City; Among of which, there are 72 large-scale enterprises and 22 enterprises with tax amount of more than a million yuan. There are more than 30,000 employees in this industry and achieve an annual output value of more than 5 billion yuan (Lou Xiumin, 2010). However, during the process of marketization and industrialization, there are serious problems of homogeneity and reproducibility in terms of the kinds of Dongyang wood carving products and serious problems of sameness in terms of the style and brand (Xu Haifeng, 2012), furthermore, the enterprises often lack awareness of brand planning and protection and thus can not effectively explore and improve the brand effectiveness of Dongyang wood carving (Ge Lisha, 2008). With the development trend of the times, people's requirements for lifestyle, consumer demand and aesthetic sentiment are increasingly demanding, and thus business competition is becoming increasingly fierce. If we can not effectively change the current situation of Dongyang wood carving products and brands, it will inevitably affect its product brand loyalty, and even get the development of Dongyang wood carving industry into a difficult position. Therefore, whether we can effectively improve the brands of Dongyang wood carving not only matters the brand loyalty of Dongyang wood carving products, but also matters the development of the entire Dongyang wood carving industry.

Attractive quality theory is a two-dimensional model of quality perception combining users' subjective feelings and product's (service) objective performance together, which is formed by introducing the two-factor theory in behavioral sciences into the field of quality management. The existing literature suggests that the brand loyalty of a product is much related to the attractive quality of the product. Based on the elaboration of the meaning of attractive quality and its relationship with creative management, the paper intends to find a way to improve the attractive quality of Dongyang wood carving and eventually improve the brand loyalty of Dongyang wood carving.

\section{Attractive Quality ANALysis of Dongyang WoOD CARVING}

American behavioral scientist Frederick Herzberg found that factors causing employees' satisfaction and dissatisfaction are different, and thus proposed the two-factor theory: factors in terms of the job itself or job content, such as challenges, recognition and responsibility from the job etc, are called incentive factors, if these conditions are met, employees will be satisfied; factors in terms of working relationships or working environment, such as, are called hygiene factors, such as salary, welfare, job security and status etc, if these conditions are not met, employees will feel dissatisfied.

Similarly, with the consumer progress, the consuming concepts of consumers are gradually changing from one-dimensional form in the past to two-dimensional form, i.e. factors that cause consumers' satisfaction and dissatisfaction 
are different. For example, as to Dongyang wood carving products, when consumer purchases a set of Dongyang wood carving mahogany sofa, if the consumer finds that the materials are impure, he will be strongly dissatisfied; however, if the materials are pure, it will not increase his satisfaction. In view of this phenomenon of consumers, Noriaki Kano (2002) proposed the KANO Model (Fig.1) to recognize quality characteristics.

KANO Model divides the quality characteristics of a product or service into five levels, i.e. essential quality characteristic, one-dimensional quality characteristic, attractive quality, indifferent quality and reverse quality, among of which, essential quality characteristic is the characteristic that customers take for granted, such as the materials of Dongyang wood carving mahogany sofa as mentioned earlier; if this kind of quality characteristic can not be met, customers will be very dissatisfied. one-dimensional quality characteristic, i.e. the quality characteristic that can meet the expectations of customers, such as whether the above-mentioned Dongyang wood carving mahogany sofa is comfortable to sit, strong and durable etc; if this kind of quality characteristic can be met, customers will be satisfied; if not, customers will be dissatisfied, and the satisfaction level is positively correlated to the contentment level. Attractive quality is the quality characteristic that satisfies the potential demands of customers, exceeds customers' expectations and brings surprise and delight to customers, such as the exquisite patters carved on the Dongyang wood carving mahogany sofa, which truly fascinate the customers; as to this kind of quality characteristic, if it is missing, it will not bring dissatisfaction to customers; however, if it is available, it will bring surprise and excitement to customers, and thus increase customers' satisfaction and improve customers' loyalty.

\section{Users' subjective feelings}

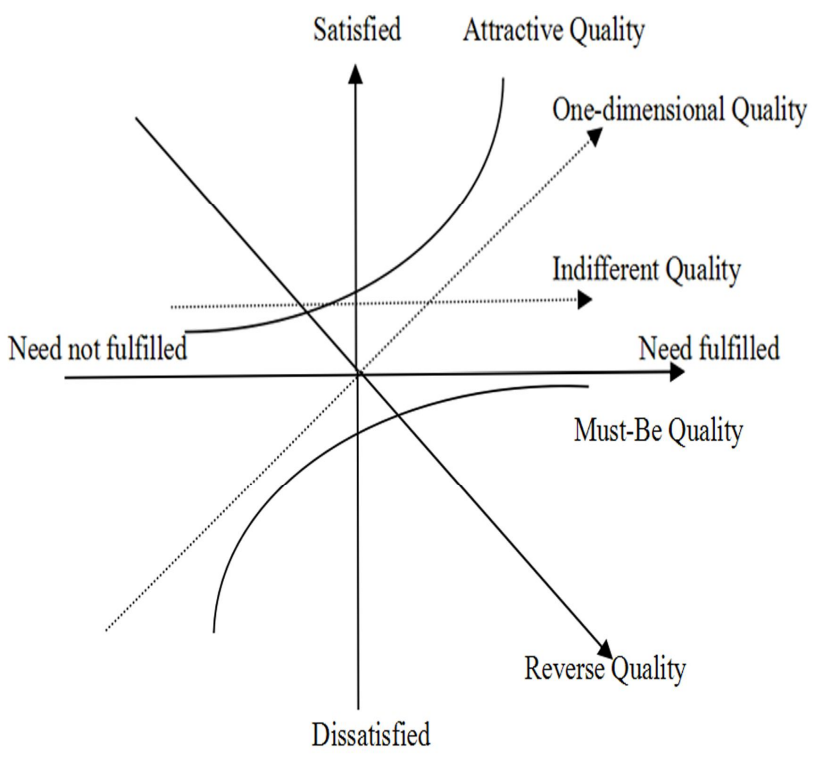

Compared to other traditional products, the biggest difference of crafts such as Dongyang wood carving is that: in addition to provide practical values for consumers, they shall also provide culture-added value for consumers by virtue of creative ideas to satisfy consumers' demand for art appreciation and finally enhance the economic value of the products. Therefore, the main indicators to measure the quality characteristics of Dongyang wood carving are not the traditional basic quality elements, such as materials, what is more important is the attractive quality. Without attractive quality indicator, the culture and arts and crafts added value implied in Dongyang wood carving can not be measured adequately. Specifically speaking, the attractive quality element of Dongyang wood carving can be reflected in the following aspects:

\section{A. Whether the subject fits the consumer demand.}

Dongyang wood carving covers a wide range of subjects, such as landscapes, human figures, natural landscapes, flower, birds, fish and insects, birds and beasts, historical themes, myths and legends, classics, emperors, folk customers, and living habits etc. However, no matter what the subject is, the key is to satisfy the aesthetic demands of people in all stations of life for Dongyang wood carving. With the economic and social development, the life concept of modern people has been changed fundamentally. The traditional craft such as Dongyang wood carving shall radiate new vigor in the new era, keep up the development rhythm of the times and represent characteristics of the times so as to fit the demands of modern people.

\section{$B$. Whether the creative concepts are skillful and orderly.}

Artistic creative concept is the subjective understanding and dynamic creation of the objective life, and contingency of subject and object and unity of subjective and objective. A skillful and orderly creative concept will enable viewers to have a unique experience and inspire their strong feeling. To accomplish this, the creator shall be able to extract deep ideas, create vivid images and truly reflect the nature of life from the raw materials in life accumulated in normal times. Therefore, the outstanding creative concepts must be the combination of emotion and reason, because emotionless works can not be truly artworks, and irrational works can not effectively express the emotions of creators.

\section{Whether the technique of expression is novel.}

Any kind of technique shall pay attention to the perfect unity of expression form and theme content. As a traditional craft, if we only focus on innovation of content but do not care about novelty of form, there will not be vitality in Dongyang wood carving and it will be impossible to obtain faster and better development in Dongyang wood carving industry. Therefore, we have to update our concepts in terms of the creative production of Dongyang wood carving and introduce modern design concept based on the integration of traditional artistic expression techniques so as to form a unique style and characteristic of Dongyang wood carving.

Fig.1. KANO Model 


\section{Creative Management And Attractive Quality of DONGYANG WOOD CARVING}

As stated above, the attractive quality of Dongyang wood carving mainly comes from the exquisite technological creation carved on Dongyang wood carving products. However, under the industrialization conditions, especially streamline processes, in addition to the factor of carving technique that affects the attractive quality of Dongyang wood carving, the most important factor is the creativity of Dongyang wood carving products. Therefore, by strengthening creative management, Dongyang wood carving enterprises can improve their creativity level in the production of Dongyang wood carving products, and thus effectively improve the attractive quality of Dongyang wood carving and then enhance the brand loyalty of Dongyang wood carving. Generally speaking, there are several ways to strengthen the creative management of Dongyang wood carving enterprises:

Firstly, creative subject management. Unlike the traditional manual production, the creative ideas in modern production of Dongyang wood carving are mainly derived from creative teams except from individuals. Therefore, a good creative idea lies, first of all, in the construction of an outstanding creative team. We shall optimize the combination of the characters and abilities of core team members, strengthen emotional ties and work incentives between them to intensify the group dynamics of members.

Secondly, creative source management. Creative ideas are traditionally derived from the research and developments of organizations. However, we should also pay attention to the creative ideas from employees of Dongyang wood carving enterprises, Dongyang wood carving experts outside the organizations and consumers. We can even carry out creative management in a comprehensive way and break the pattern of isolated creation entirely by the research and development department to improve the creative management level of enterprises.

Thirdly, creative cultural management. Some world renowned enterprises are filled with creative culture and atmosphere therein, which plays an important role in inspiring and stimulating employees' creativity and giving their talents to full play. \An enterprise, which is good at creative cultural management, will often use a creative vision, internal cultural communication within the organization and an effective incentive mechanism to build its own creative cultural atmosphere.

\section{CONCLUSION}

Attractive quality is the key to improve the market competitiveness of Dongyang wood carving. Under the conditions of industrialization of Dongyang wood carving industry, especially against the background of streamline production of wood carving, only by strengthening the creative management and improving the creativity level of Dongyang wood carving, can we effectively improve the attractive quality of Dongyang wood carving and then enhance the brand loyalty of Dongyang wood carving to put Dongyang wood carving in an invincible position in the fierce market competition.

\section{REFERENCES}

[1] Ge Lisha, Research on the Design Strategy in the Brand Improvement of Dongyang Wood Carving, MA Thesis, Shanghai Jiaotong University, 2001 (01).

[2] Yuan Weiwei, A Primary Discussion on the Elements and Methods of Creative Management, Managers, 2012(12).

[3 Xu Haifeng, Thinking and Analysis of Specialized Market on the Development of Dongyang Wood Carving Cultural and Creative Industry, Hua Zhang, 2012(03).

[4] Gu Fangsong, Crisis Faced by Dongyang Wood Carving Art, Zhejiang Arts and Crafts, 1998 (04).

[5] Huang Xiaoming, A Primary Discussion on the Comprehensive Application of Traditional Technique and Modern Concept of Dongyang Wood Carving, Sculpture, 2006 (02).

[6] He Meifang, On the Innovation of Design of Dongyang Wood Carving, Zhejiang Arts and Crafts, 2009 (04).

[7] Lin Shu, Composition and Significance of Dongyang Wood Carving Patterns, Literature and Art Studies, 2009 (01).

[8] Huang Xiaoming, Application of Dongyang Wood Carving in Modern Decoration, Sculpture, 2011 (03).

[9] Wang Qin, Origin and Decorative Art Characteristics of Dongyang Wood Carving, Art Panorama, 2012 (02).

[10] Zhang Lili, Loss and Development of Regional Art and Culture —A Case Study of Zhejiang Dongyang Wood Carving, , CSTA Forum, 2009 (05).

[11] Jin Bosong, Regional Cultural Edge of Dongyang Wood Carving, Zhejiang Arts and Crafts, 2007 (03).

[12] Jin Bosong, Cultural and Historical Origin of Dongyang Wood Carving, Shanghai Arts and Crafts, 2009 (02).

[13] Zhu Jun, Current Status and Conservation of Zhejiang Dongyang Wood Carving in Ming Dynasty, Journal of Zhejiang Vocational Academy of Art, 2010 (12) 